\title{
Bias signal control in differential protection
}

\author{
Viktor Kuprienko ${ }^{1, *}$ \\ ${ }^{1}$ Orenburg State University, 460018, Pobedy Ave., 13, Orenburg, Russia
}

\begin{abstract}
The problem is to ensure the stability of the differential protection functioning at deep saturation of the cores of electromagnetic current transformers. The errors of current transformers support the greatest influence on the operation of differential protections. Features of the differential protection operation at deep saturation of current transformers in short-circuit transient modes are considered. Comparison results of various algorithms for the formation of a bias signal are given. The control capability of the bias signal generation algorithm was analyzed. The harmonic composition of the differential current on the mathematical model of twoarm differential protection in the transient mode with external and internal short circuits at saturation of the current transformers of one arm was investigated. The stability of the differential protection functioning can be enhanced by the selectivity of the bias signal generation algorithm. A bias signal control criterion using a relative level of higher harmonics to a differential signal was proposed.
\end{abstract}

\section{Introduction}

Biased differential protection (BDP) is widely used at various power supply facilities. It is known that the parameters characterizing the nonoperation of BDP with external and sensibility with internal short-circuiting (SC) depend on the type of bias signal (BS). Also, various algorithms for the formation of BS are known.

\section{Problem Statement}

Ensuring the selectivity and operation stability of the differential protection with the saturated core of current transformers (CT) in transient modes is often a problem. Errors of the CT have the greatest impact on the operation of differential protections, and in particular - the protection of transformers.

\section{Research Questions}

The application of bias improves the indicators of differential protection. Some manufacturers of microprocessor devices BDP use a relay bias characteristic in the form of a

\footnotetext{
*Corresponding author: prigozhins@ mail.ru
} 
broken line with two transitions to different bias coefficients $\left(\boldsymbol{C}_{\boldsymbol{b}}\right)$. In «Schneider Electric» it was called «self-adapting bias».

According to the method developed by the author, a comparative analysis of BDP with different types of BS was conducted [1]. As a result, it was concluded that among the known DS there is no universal one, that would ensure a stable functioning of the protection in all modes of operation taking into account the load current and the phase displacement of the side currents.

\section{Purpose of the Study}

The BDP's operation stability can be increased if each BS would only work in those regimes in which his worth are shown, i.e. it seems advisable to use several BS and their automatic selection depending on the working conditions of the protection. Not only a change in $\boldsymbol{C}_{\boldsymbol{b}}$, but a change in the algorithm itself for the formation of the BS. The principle of «selective bias» was proposed by the author at the turn of the 1990-s [2] and implemented on an analogous element base.

The use of microprocessors opens up new possibilities. When investigating the properties of BDP that are manifested when working with a CT with errors due to saturated core, it is expedient to analyze the harmonic composition of the differential current. In this mode, in the secondary current of the CT, the aperiodic component and all higher harmonics are present and besides the amplitudes of the last ones depend on the parameters of the regime and are functions of time.

The signal due to higher harmonics $(\boldsymbol{G})$ can be used to correct the operation of the BDP. In the well-known works studying biasing of higher harmonics, the use of this signal did not give the desired effect, since the level of the higher harmonics of the differential current is practically independent of the location of the SC (external or internal).

The search for an algorithm of the functioning of BDP has importance in connection with the foregoing, allowing to increase the level of nonoperation of protection for external SC, accompanied by saturated core of CT. The analysis of the correlation of signals is of interest - main (differential and bias) and auxiliary (using the level of higher harmonics $\boldsymbol{G}$ of the differential current).

\section{Research Methods}

Information on the shape of the differential current can be used as a criterion for saturated core of the CT. The author conducted researches using the «Matlab Simulink» harmonic composition of the differential current on the mathematical model of a two-side BDP in a transient behavior with external and internal SC at saturated core of the CT of one side. The determination of the higher harmonics level $\mathbf{G}$ of the differential current was produced for each period by the expansion in a Fourier series.

Calculations on the mathematical model of BDP were carried out using the following initial data: CT load is accepted as maximum permissible by the condition of $10 \%$ error, cos $\varphi=0.8$; mismatch sides currents of protection $17 \%$; the time constant of the damping of the aperiodic component of the current SC $\boldsymbol{T}_{\boldsymbol{a}}=0.05$ and 0.3 seconds; the range of variation of the current multiplicities SC $1 \div 20$.

According to calculations, the level of the higher harmonics $(\boldsymbol{G})$ increases to $\boldsymbol{\varepsilon} \approx 50 \%$ and then falls (figure 01). Reliability and general character of the results, obtained for a particular type of CT, were confirmed using a generalized parameter $\boldsymbol{A}_{\boldsymbol{Z}}$ [3] (Fig. 1). 


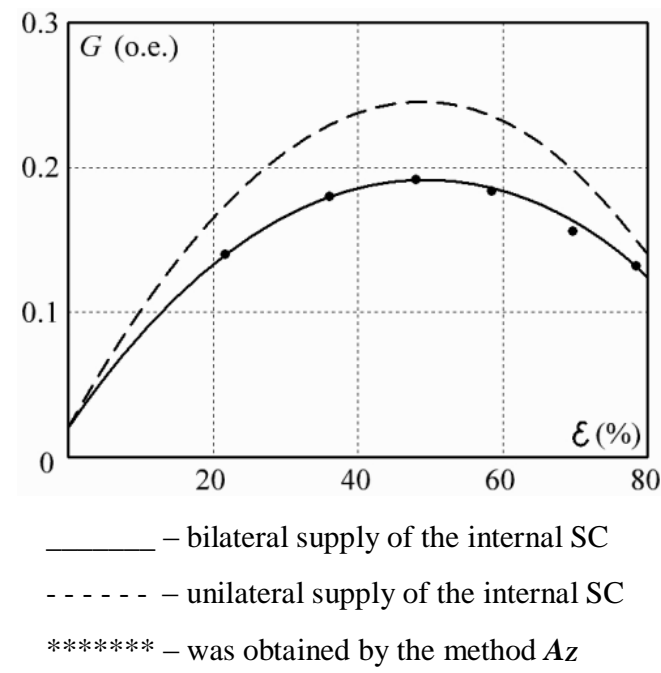

Fig. 1. Dependencies of the higher harmonics level of the differential current on the total error of CT.

\section{Findings}

The dependence of the higher harmonics level $\boldsymbol{G}$ of the differential signal from $\boldsymbol{A}_{\boldsymbol{Z}}$ is shown in Fig. 2. The values of $\boldsymbol{G}$ are given in relative units, the level of the differential signal is taken as the basis, corresponding to rated current $\boldsymbol{C}_{\boldsymbol{r}}$.

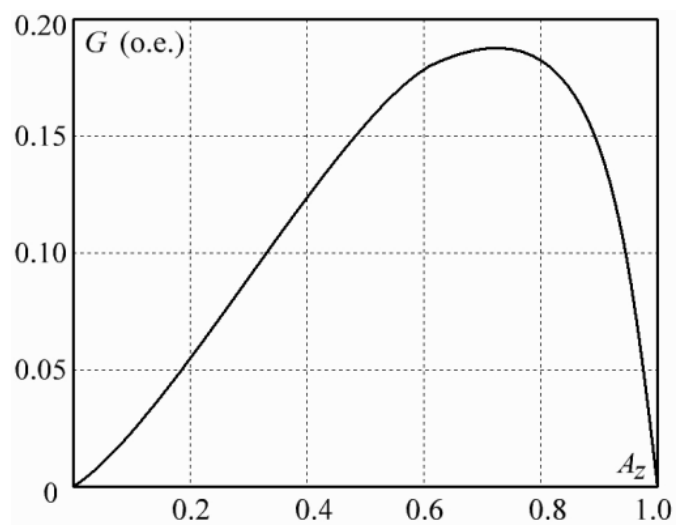

Fig. 2. Dependencies of the higher harmonics level of the differential current on the generalized parameter $\boldsymbol{A} z$.

When the CT is saturated, the value of $\boldsymbol{G}$ for the external and internal SC is practically the same. That is, $\boldsymbol{G}$ as a criterion for the degree of saturation of CT also has limited possibilities (for example, $\boldsymbol{G}$ is the same for $\varepsilon=20$ and $75 \%-$ Fig. 1).

The relative level of the higher harmonics $G^{*}$ is more suitable for this purpose - a quantity proportional to the ratio of $\boldsymbol{G}$ to the differential current: 


$$
G^{*}=\frac{G}{I_{d}}
$$

The dependencies $G^{*}(\varepsilon)$ for the initial period of the emergence of the SC are shown in Fig. 3.

The type of graphs does not depend on the multiplicity of currents of SC, time and is practically stable over a wide range of values of the aperiodic component of the current SC $\boldsymbol{T}_{\boldsymbol{a}}$. The essential difference between $\boldsymbol{G}^{*}$ with external and internal and SC is a positive quality.

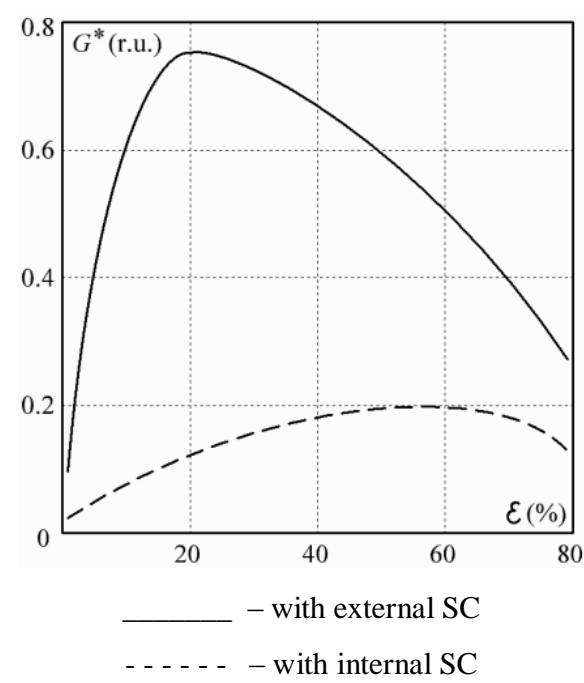

Fig. 3. Dependencies of the relative value of the higher harmonics level of the differential current on the total error of the CT.

Analysis of these dependencies allows to conclude that the value of $G^{*}$ can be expediently used to switch the BS of the differential protection. This fulfillment of the BDP, when the BS is selected depending on $G^{*}$, allows to significantly eliminate the contradiction in ensuring selectivity and sensitivity.

\section{Conclusion}

The stability of BDP functioning can be increased by selectivity of the BS formation algorithm.

As a criterion controlling the choice of the BS, it is advisable to use the relative level of the higher harmonics (with respect to the differential signal).

\section{References}

1. V. V. Kuprienko, European Science Review, 2 (2014)

2. A. N. Burnashev, V. V. Kuprienko, Device for differential protection of electrical installation. Patent for invention RUS 2024145 (1994) 
3. V. E. Kazansky, Current transformers in relay protection systems (Energia, Moscow, 1978) 